\title{
Vibration health monitoring of rolling bearings under variable speed conditions by novel demodulation technique
}

\author{
Dezun Zhao ${ }^{\mathrm{a}}$, Len Gelman ${ }^{\mathrm{a}}$, Fulei Chu ${ }^{\mathrm{b}}$, Andrew Ball ${ }^{\mathrm{a}}$ \\ ${ }^{a}$ Centre for Efficiency and Performance Engineering (CEPE), School of Computing and Engineering, University of \\ Huddersfield, Huddersfield, UK \\ ${ }^{\mathrm{b}}$ Department of Mechanical Engineering, Tsinghua University, Beijing 100084, China
}

\begin{abstract}
Time-varying fault impulse amplitude and time-varying fault impulse interval are the main challenges for rolling bearing fault diagnosis under variable speed conditions. In this paper, a vibration based rolling bearing fault characteristic frequency (FCF) estimation and novel diagnosis method under time-varying rotational speeds are proposed, using the demodulation transform. Firstly, the FCFs with weak amplitudes are estimated, using the optimization based demodulation transform, whose main concept is, that with the optimal demodulation operator (DO), the time-varying frequency component can be transformed into a constant one and the largest peak can be detected in the spectrum of the demodulated signal. Secondly, the rolling bearing is successfully diagnosed with hypothesis based demodulation transform, whose main concept is, that the rotational frequency (RF) -related DOs under different fault types are estimated, based on the estimated FCFs and the fault characteristic coefficients (FCCs). Then the RF-related peak in the spectrum of the demodulated signal can be used to detect rolling bearing fault. The effectiveness of the proposed method is verified, using both simulated signal and measured vibration signal. Novel comparisons with the current methods demonstrate much better ability of the proposed method for diagnosis of rolling bearing under variable speed conditions.
\end{abstract}

Keywords: Rolling element bearing; Vibration health monitoring; Signal processing; Variable rotational speed; Demodulation transform.

\begin{tabular}{|c|c|c|c|}
\hline \multicolumn{4}{|c|}{ Nomenclature } \\
\hline FCF & Fault characteristic frequency & SNR & Signal-to-noise ratio \\
\hline DO & Demodulation operator & IF & Instantaneous frequency \\
\hline FCC & Fault characteristic coefficient & AM-FM & $\begin{array}{l}\text { Amplitude modulation and } \\
\text { frequency modulation }\end{array}$ \\
\hline RF & Rotational frequency & STFT & Short-time Fourier transform \\
\hline FFT & Fast Fourier transform & WT & Wavelet transform \\
\hline $\mathrm{COA}$ & Computed order analysis & SST & Synchrosqueezing transform \\
\hline GDT & Generalized demodulation transform & SET & Synchroextracting transform \\
\hline TFA & Time-frequency analysis & TFR & Time-frequency representation \\
\hline
\end{tabular}

\section{Introduction}

The rolling bearing is one of the most widely used mechanical parts in rotating machinery $[1][2][3][4][5][6]$. Because of tough working conditions, rolling element bearings are usually subjected to a local damage, such as a crack, a pitting, et al. Rolling bearing fault is one of the major reasons for a shutdown of machines. For reducing economic losses and avoiding accident, vibration based rolling bearing 
fault diagnosis has become an important topic in both academic and industrial areas for several decades [7][8][9][10].

When a bearing has a local defect, the contact between a defect and the mating surface will introduce transient impulses to the vibration response of the bearing system [11][12]. The repetition frequency of the impulses is named the FCF, whose ratio to the RF is a constant and different FCF-to RF ratios for a specific bearing, which are named FCC, represent different fault types [13]. In engineering, the rolling bearing often works under time-varying speed conditions such as start-up, shut-down [14] [15] [16] and RF changes during machine operation.

Comparted with constant speed conditions, there are two differences for fault bearing signal under variable speed conditions. First, the repetition frequency of the transient impulses is time-varying, which will bring spectral smearing phenomenon in the fast Fourier transform (FFT)-based spectrum. Second, the impulse amplitude also changes with the time, i.e., under a low speed, the impulse amplitude is small, and the impulse amplitude is relatively large under a high rotational speed [17]. As a result, the FCF-related time-frequency ridges are easy to be overwhelmed by noise.

For bearing fault diagnosis under time-varying rotational speeds, there are a lot of effective methods, which can be divided into three categories: (a) the computed order analysis (COA) [18] [19]based methods; (b) the generalized demodulation transform (GDT) [20][21] based methods; and (c) the time-frequency analysis (TFA) [22][23] based methods.

In the COA, the time domain time-varying signal is transformed into angular domain stationary signal with the resampling. Based on the COA, Guo proposed an envelope order tracking method which is effective for bearing nonstationary fault feature extraction [24]. In the proposed method, the fast kurtogram algorithm is applied to remove noise, then the envelope of the filtered signal is resampled, and lastly the envelope order spectrum is estimated for bearing fault detection.

For avoiding the RF measurement, the FCF extraction based COA was developed by Wang [13]. The extracted FCF is used to resample the faulty bearing signal and fault type is determined by the envelope order spectrum. In addition, for addressing the shortcomings of the traditional COA and further reducing the computational burden, Wang proposed a tacho-less order tracking method based on the order spectrogram visualization [25]. However, it should be noted that data interpolation is the main process of the COA, and the interpolation errors cannot be neglected in the process of bearing fault detection [26][27].

TFA can be used to characterize time-varying fault components of nonstationary signals. Hence, TFA also has also been widely used for bearing nonstationary fault feature detection. The generalized synchrosqueezing transform (SST) [28] is employed to low frequency band and the resonance band of rolling bearing for enhancing the fault-related time-frequency ridges and then RF-related spectrum and FCF-related spectrum are obtained to detect bearing fault.

Yu [29] developed a novel TFA method, the transient-extracting transform, which can effectively extract the transient components in the rolling bearing signals under variable speed conditions. Shi [30] proposed an iterative generalized stepwise demodulation transform to improve the energy concentration level and the proposed method is successfully applied to rolling bearing fault diagnosis under time-varying speed. However, it should be noted, that time-varying FCF cannot be quantitatively characterized by TFR for detecting bearing fault in essentially noisy environment. 
The GDT is an effective method for fault detection of rolling bearing under time-varying speeds. The main idea of the GDT is that the time-varying frequency curve can be transformed into a constant curve, based on the DO [31][32]. Based on the GDT, a compound fault diagnosis method for rolling bearing was proposed by Zhao [33]. The FCF-related DOs are estimated [33], based on the measured RF, and bearing faults are detected, using the spectra of demodulated signals. In engineering applications, the RF measurement devices are not easily installed due to limitation of space and cost. Hence, the proposed bearing fault diagnosis method without the RF measurement device is important.

For avoiding RF measurement, a high-resolution TFA is introduced to the GDT- based bearing fault diagnosis method [34], in which the FCF is extracted, using the peak searching algorithm. The windowed fractal dimension transform is used [35] to pre-process raw signal for extracting FCF and the GDT is applied for bearing fault diagnosis. Liu [36] proposed an iterative GDT for processing fault related harmonics of rolling bearing. Zhao [37] developed an optimized GDT for rolling bearing fault diagnosis in nonstationary conditions. In this method, the raw signal with a clear fault feature is selected for demodulation transform and bearing type is detected.

The FCF in Refs [34] - [37] are not fully polluted by noise and, therefore, could be easily extracted based on TFA and peak searching method. Considering time-varying impulse amplitude, the FCFs cannot be easily extracted by advanced TFA. The main reason is that, normally, fault related impulse amplitude changes with RF: i.e. under a low RF, the impulse amplitudes are weak compared with impulse amplitudes under a high RF. As a result, the FCFs in TFR are relatively weak and easily polluted by noise. Hence, the above methods cannot work well in noisy environment.

Based on the above analysis, the most of current papers are focusing on time-varying fault impulse interval and are not effective for time-varying fault impulse amplitude. Due to the time-varying fault impulse amplitude, the FCF cannot be fully extracted from TFR by the peak searching method, even the TFR is estimated with a high-resolution. Hence, the following problem should be considered for bearing fault diagnosis under time-varying speeds: development of new technologies, that can effectively diagnose bearing faults in conditions of time-varying impulse amplitudes and time varying impulse intervals, related to faults. This problem is especially important in conditions of low rotational speed.

Therefore, the main objectives of this paper are to

(a) develop a novel rolling bearing diagnosis technique for conditions of time-varying impulse amplitudes and time varying impulse intervals, related to bearing faults.

(b) validate the proposed technique by both simulations and experiments under time-varying rotational speeds.

(c) perform a novel comparison of the proposed technique with the traditional GDT-based bearing fault diagnosis and other TFA-based bearing fault diagnosis methods.

(d) perform a novel parameter optimization of the proposed technique in conditions of time-varying impulse amplitudes and time varying impulse intervals, related to bearing faults.

The main novelty of the paper is a novel diagnostic technique, that is a combination of optimization approach and the GDT for conditions of variable fault related impulse amplitudes. Our intensive literature 
search has shown, that nobody used such a novel combination of optimization approach and the GDT for correctly estimating FCF in conditions of variable impulse amplitudes, related to bearing faults. Specific novelties are as follows:

(a) with the optimization based demodulation transform, the FCF, that overwhelmed by noise, can be accurately estimated. The main concept is that optimization approach is used for circulated estimation of FCF related demodulation operator (DO) until a largest peak can be captured in the spectrum of the demodulated signal. With the optimal DO, the time-varying FCF is transformed into a constant one by the demodulation transform, and the corresponding peak is largest in the spectrum of the demodulated signal.

(b) with hypothesis based demodulation transform, the rolling bearings can be quantitatively diagnosed. Assuming, that the estimated FCF is induced by the outer race fault, the RF related DO can be estimated by the FCC of outer race and the FCF. The corresponding spectral peak is obtained from the spectrum of the demodulated signal, which is obtained by the demodulation transform and the FFT.

The comparison between the estimated peak frequency and the theoretical peak frequency can be used to quantitatively detect bearing fault. Similar assumptions could be made for inner race and rolling element faults. The proposed technology is a novel generalisation of the GDT in non-stationary conditions of variable impulse amplitudes, related to faults. It will make an influence on health monitoring of rotating machinery and structures in non-stationary conditions.

Hereinafter, the paper is organized as follows: In Section 2, the novel technique is introduced, and a demonstration is presented. Then, the rolling bearing fault diagnosis method, using hypothesis based demodulation transform, is described in Section 3. In Section 4, a bearing signal, related to a local fault, under time-varying rotational speed is simulated to validate the effectiveness of the proposed diagnosis technique. In Section 5, measured bearing signal under variable speed is analysed, and five novel comparisons are discussed for further validation of the proposed technique. Finally, the conclusions are drawn in Section 6.

\section{IF estimation method, using optimization based demodulation transform}

In this section, the IF estimation method, using optimization based demodulation transform, is introduced, and then is validated by a simulation.

\subsection{Optimization based demodulation transform}

The main concept of the optimization based demodulation transform is to introduce the optimization algorithm to the GDT for estimating an IF curve.

An AM-FM nonstationary signal, shown in Eq. (1), is considered:

$$
x(t)=A(t) \cos \left(2 \pi \int_{0}^{t} f(s) d s\right)
$$

where $A(t)>0$ is the instantaneous amplitude; $f(s)$ is the IF. It is assumed that $f(s)=a_{1}+b_{1} s+$ $c_{1} s^{2}>0$, where $a_{1}, b_{1}$ and $c_{1}$ denote the initial frequency, the chirp rate and the frequency acceleration, respectively. Different orders for IF functions can be selected under different cases.

The analytic signal of Eq. (1) is estimated as

$$
x_{A}(t)=x(t)+j \mathrm{H}(x(t)) \approx A(t) \exp \left(j 2 \pi\left(a_{1} t+\frac{1}{2} b_{1} t^{2}+\frac{1}{3} c_{1} t^{3}\right)\right)
$$


where $H(\bullet)$ is operator of the Hilbert transform.

The DO of the analytic signal is defined as

$$
\Phi\left(t ; b, c, t_{r}\right)=\exp \left(-j 2 \pi\left(\frac{b}{2}\left(t^{2}-2 t_{r} t\right)+\frac{c}{3}\left(t^{3}-3 t_{r}^{2} t\right)\right)\right)
$$

where $b$ and $c$ are the demodulation parameters and $t_{r} \in \mathbb{R}$ is reference time.

The demodulated signal is estimated as:

$$
\begin{gathered}
x_{d}\left(t ; b, c, t_{r}\right)=x_{A}(t) \Phi\left(t ; b, c, t_{r}\right)= \\
A(t) \exp \left(j 2 \pi\left(a_{1} t+b t_{r} t+c t_{r}^{2} t+\frac{1}{2}\left(b_{1}-b\right) t^{2}+\frac{1}{3}\left(c_{1}-c\right) t^{3}\right)\right)
\end{gathered}
$$

The IF of the demodulated signal is $f_{d}(t)=\left(c_{1}-c\right) t^{2}+\left(b_{1}-b\right) t+a_{1}+b t_{r}+c t_{r}^{2}$. Let $c_{1}=c$ and $b_{1}=b$, the demodulated signal will become a purely AM signal and will concentrate on frequency $f=$ $a_{1}+b t_{r}+c t_{r}^{2}$, which is the IF of signal $x_{A}(t)$ at $t_{r}$. When a suitable DO is applied to the analytic signal, a clear peak can be captured in the spectrum of the demodulated signal. Positive frequency part of the spectrum is estimated as

$$
F\left(t ; b, c, t_{r}\right)=\frac{1}{2 \pi} \int_{0}^{\infty} x_{d}\left(t ; b, c, t_{r}\right) \exp (-j \omega t) d t
$$

Peak of the spectrum is estimated as

$$
p(b, c)=\arg \underset{f}{\max }\left(\left|F\left(f ; b, c, t_{r}\right)\right|\right)
$$

where $\arg \max (\bullet)$ represents argument of function maximum.

The chirp rate and the frequency acceleration of the raw signal are estimated by optimizing the spectrum as

$$
\left\{\widetilde{b_{1}}, \widetilde{c_{1}}\right\}=\underset{b, c}{\arg \max }(p(b, c))
$$

Eq. (7) can be addressed, based on various optimization algorithms. Due to its advantages, the particle swarm optimization is selected for optimization task [38]. The population size and the maximum iteration are set to 60 and 600, respectively. The order of the DO can be determined by the local IF feature in TFR of target signal, which is introduced in section 2.3.

The initial frequency is estimated as

$$
\widetilde{a_{1}}=\arg \max _{f}\left(\left|F\left(f ; \widetilde{b_{1}}, \widetilde{c_{1}}, t_{r}\right)\right|\right)-\widetilde{b_{1}} t_{r}-\widetilde{c_{1}} t_{r}^{2}
$$

\subsection{Local IF curve model}

For IF estimation, local IF curve model is determined, based on the time-frequency feature of signal $x_{A}(t)$. TFR is estimated, based on the STFT, as

$$
S(t, f)=\int_{-\infty}^{+\infty} g(\tau-t) x_{A}(\tau) e^{-i 2 \pi f \tau} d \tau
$$

A peak searching algorithm, Eq. (10), is employed to extract IF from the STFT $S(t, f)$. For improving the robustness against noise, the local maxima detection approach is employed, as follows: 


$$
\left\{\begin{array}{c}
\left(t_{s}, I F\left(t_{s}\right)\right)=\arg \max _{f, t}[S(t, f)] \\
I F\left(t_{i}\right)=\arg \max _{f}\left[S\left(t_{i}, f\right)\right]
\end{array}\right.
$$

where $\left(t_{s}, I F\left(t_{s}\right)\right)$ is a time-frequency position of maximum STFT amplitude, obtained via maximization of the STFT with respect to time and frequency, $t_{s}$ is a time position of maximum STFT amplitude, $\operatorname{IF}\left(t_{s}\right)$ is a frequency position of maximum STFT amplitude and it is an estimate of the IF at a time position $t_{s}$; in second line, the algorithm searches in frequency range $\left[I F\left(t_{i-1}\right)-\frac{\Delta p}{2}<f<I F\left(t_{i-1}\right)+\frac{\Delta p}{2}\right]$ at time $t_{i}, i=1$, $2, \cdots I, I$ is determined by time resolution of the STFT and length of the signal, $\Delta p$ is a local searching frequency range, $t_{0}=t_{s}$; time variation $t_{i}=t_{i-1}+\Delta t$ is used for the forward searching, time variation $t_{i}=t_{i-1}-\Delta t$ is used for the backward searching, $0 \leq t_{i} \leq T, T$ is time duration of a raw signal, $\Delta t$ is time resolution of the STFT, $\operatorname{IF}\left(t_{i}\right)$ are estimates of the IF: i.e. multiple searching results, obtained via maximization of the STFT with respect to frequency at the selected times.

The peak searching algorithm (10) is based on an iterative approach to determine the IF: it starts from the maximum of the STFT amplitude and traces IF forward and backward in time.

The extracted IFs have errors at low rotational speed due to noise interference. Based on number of continuous time interval, where the IF cannot be accurately extracted, signal $x_{A}(t)$, whose time duration is $[0, \mathrm{~T}]$, is divided into $k$ intervals. In general, IF curves of bearing signal are not complex. Hence, $k$ is set to 2 and 3: i.e. case 1 and case 2, as shown in Fig.1, and described as

$$
\begin{gathered}
\text { Case 1: }\left\{\begin{array}{l}
I_{1}=\left[0, T_{e}\right] \\
I_{2}=\left[T_{e}, T\right]
\end{array}\right. \\
\text { Case 2: }\left\{\begin{array}{l}
I_{1}=\left[0, T_{s}\right] \\
I_{2}=\left[T_{s}, T_{e}\right] \\
I_{3}=\left[T_{e}, T\right]
\end{array}\right.
\end{gathered}
$$

where $T_{s}$ is starting point and $T_{e}$ is ending point.

The following points should be noted: (a) number $k$ should be set to higher number, when RF curve is very complex, i.e., more interval points $\left[T_{s}, \cdots T_{i}, \cdots, T_{e}\right]$ should be determined and the local model should be estimated, based on Eqs. (11) and (12); (b) one of IF curves in every two adjacent intervals should be accurately estimated, using Eq. (10). 

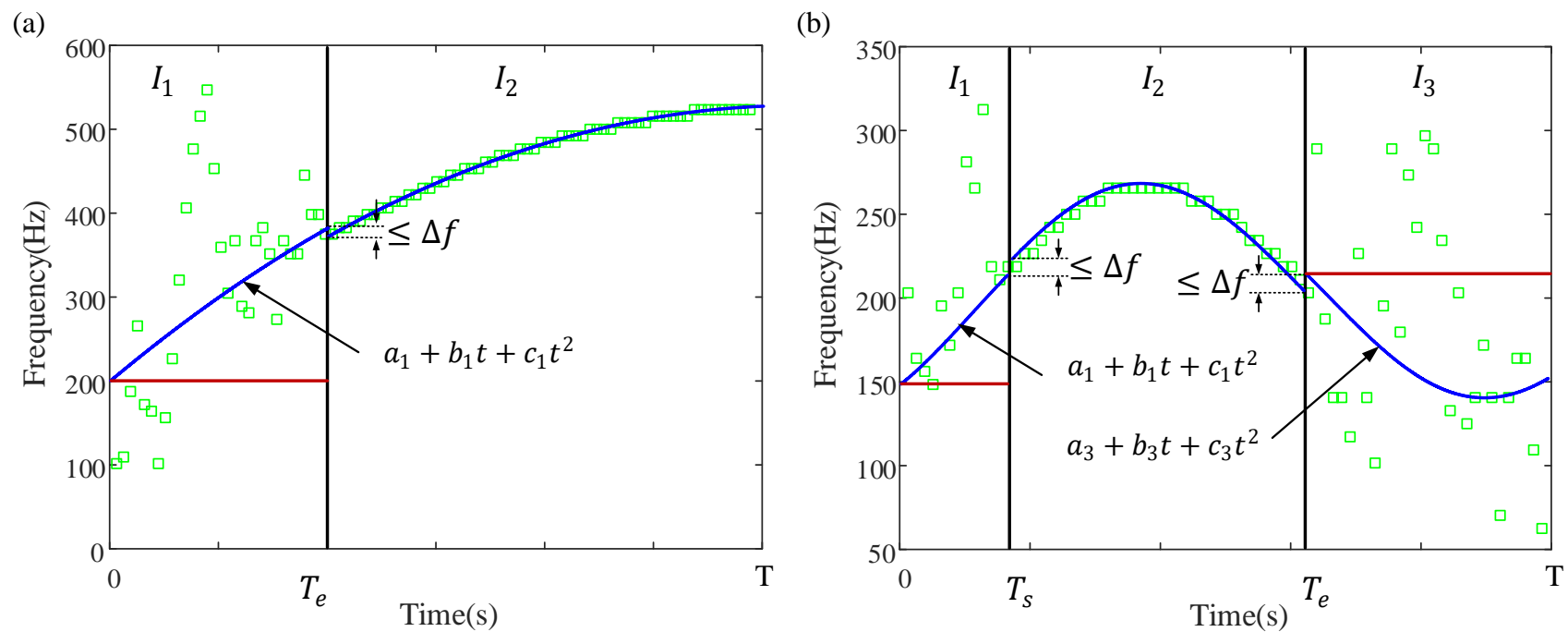

Fig.1 Illustration of optimization based demodulation transform: (a) case 1 (b) case 2

The accuracy/effectiveness of the method is increasing with increase of a polynomial order, used for local approximation, and number of time intervals. However, the computational complexity is also increasing with increase of a polynomial order and number of intervals. The required polynomial order and number of time intervals depends on the complexity of nonstationary IF curve for RF and FCF. It is hard to make a decision on these numbers, based on the initial TFR of the envelope, as all initially extracted IFs are, normally, partially polluted by noise.

Therefore, an iterative procedure is employed in the proposed method for both number of intervals and a polynomial order. For the first iteration of IF estimation/fault diagnosis, number of intervals is always 2 and a polynomial order is also always 2 . The obtained error, related to these parameters, is comparing with a termination threshold $\Delta f$ (Eq. (13)) and if an error is less, than a termination threshold, the estimation/diagnosis is finalised, using results of the first iteration.

If the obtained error is more, than a termination threshold, the number of intervals is increased by 1 and a polynomial order is also increased by 1 and method is performing the second iteration with three intervals and with a polynomial order of three. The obtained error, related to these parameters, is comparing with a termination threshold and if an error is less, than a termination threshold, the estimation/diagnosis is finalised, using results of the second iteration.

If the obtained error is more than a termination threshold, the number of intervals is again increased by 1 and a polynomial order is also increased by 1 and method is performing the third iteration with four intervals and with a polynomial order of four and so on.

\subsection{IF estimation}

IF curve, in every time interval, where the IFs cannot be accurately extracted, is estimated using the optimization based demodulation transform. 
The local signal $\left.x_{A}(t)\right|_{t \in I_{i}}(i \in[1,2,3])$ time interval $I_{i}$ can be expressed, using Eq. (2), with the assumption, that variation of the IF curve is simple, i.e. phase order of the analytic signal is equal to 2 , which is close to the real IF curve. Based on Eq. (3), a DO of time interval $I_{i}, \Phi_{I_{i}}\left(t ; b, c, t_{r}\right)$, is determined.

The corresponding demodulated signal $\left.x_{d}(t)\right|_{t \in I_{i}}\left(t ; b, c, t_{r}\right)$ is estimated using Eq. (4). Then, positive part of the spectrum of the demodulated signal $F\left(t ; b, c, t_{r}\right)$ is estimated, based on Eq. (5).

Based on Eq. (4), the time-varying IF curve is transformed into a constant line (red line in Fig.1) when the optimal DO is estimated, i.e. $b_{i}=b$ and $c_{i}=c$. As a result, a clear peak $P_{I_{i}}(b, c)$ is captured in the spectrum using Eq. (6).

Using Eq. (7) the IF parameters $\left\{\widetilde{b_{l}}, \widetilde{c_{l}}\right\}$ are estimated. Finally, the initial frequency is estimated, using Eq. (8).

For forming a continuous curve, $\widetilde{a_{l}}$ in Eq. (8) should be satisfied as follows.

$$
\left|\widetilde{I F}_{I_{i}}^{s}-\widetilde{I F}_{I_{i-1}}^{e}\right| \leq \Delta f
$$

where $i$ is equal to 2 and $3, I F_{I_{i}}^{s}$ is starting frequency of time interval $I_{i}$, and $I F_{I_{i-1}}^{e}$ is ending frequency of time interval $I_{i-1}$, from which, one frequency point can be calculated based on $\widetilde{a_{l}}, \widetilde{b_{l}}$ and $\widetilde{c_{l}}$, another can be accurately extracted.

The signal is divided into time intervals and it is needed to estimate IF in every time interval. From the obtained IF curve in time interval $I_{i}$, it is possible to get its starting frequency $\widetilde{I F}_{I_{i}}^{S}$ and ending frequency $\widetilde{I F}_{I_{i}}^{e}$. Eq. (13) means, that the starting frequency $\widetilde{I F}_{I_{i}}^{s}$ and ending frequency $\widetilde{I F}_{I_{i-1}}^{e}$ in the previous time interval $\left(I_{i-1}\right)$ should have small difference $\Delta f$. $\Delta f$ should be selected relatively small to reduce estimation error of the particle swarm optimization. For getting precise results and decreasing computing time, searching regions for the chirp rate $b$ and the frequency acceleration c are set to [-100 100] and to [-6 6] respectively and $\Delta f$ is set to $10 \mathrm{~Hz}$.

An error is defined for the estimated IF curve as

$$
R E=\max \left(\frac{|I F(t)-\widetilde{I F}(t)|}{I F(t)}\right)
$$

where $\widetilde{I F}(t)$ and $I F(t)$ denote the estimated IF curve and the real IF curve, respectively.

One of the main advantages of the proposed method is, that it is not required a priori knowledge or a measurement of a rotation speed. Eq. (14) is used to evaluate effectiveness of the proposed method for calculating an IF curve. Hence, the known real IF curve is used here only for estimation of effectiveness of the proposed method. In engineering applications, that are not providing a priori knowledge or a measurement of a rotation speed, it is not possible to calculate an estimation error. In engineering applications, that are providing a priori knowledge or a measurement of a rotation speed, it is easy to evaluate an IF and it is, normally, not needed to calculate an estimation error.

The proposed method also can be updated by iteration for processing multiple IF curves.

\subsection{Demonstration}

For validating the effectiveness of the IF estimation algorithm, a simulated AM-FM signal $x(t)$ with the white Gaussian noise is constructed as

$$
\left\{\begin{array}{c}
f(t)=50(\sin (0.45 \pi t)-\cos (0.38 \pi t))+200 \\
A(t)=100 e^{-2(t-2)^{2}}+1 \\
x(t)=A(t) \sin \left(2 \pi \int_{0}^{t} f(s) d s\right)+n(t)
\end{array}\right.
$$


Signal duration is $5 \mathrm{~s}$; the sampling frequency is $2000 \mathrm{~Hz}$; the signal to noise ratio (SNR) is $-25 \mathrm{~dB}$. The IF curve and the simulated signal are presented in Fig.2 (a) and (b), respectively. Fig.2 (c) is TFR of the simulated signal. From TFR, it can be found, that the time-frequency ridge is completely overwhelmed by noise in zoom 1 and zoom 2. The IFs in zoom 1 and zoom 2 cannot be accurately extracted from TFR, as shown in Fig. 3 (a). In this case, the signal is divided into 3 parts and local model can be determined by Eq. (12), where $T_{s}=0.8$ and $T_{e}=3.1$. Hence, $0 \leq I_{1}<0.8,0.8 \leq I_{2}<3.1$ and $3.1 \leq I_{3} \leq 5$. Based on the proposed algorithm, the optimal IF curve functions in every parts can be estimated as

$$
\begin{gathered}
\left.f(t)\right|_{t \in I_{1}}=149+79.9 t+0 t^{2} \\
\left.f(t)\right|_{t \in I_{2}}=107.7+164.3 t-42.8 t^{2} \\
\left.f(t)\right|_{t \in I_{3}}=890.5-334.5 t-37.3 t^{2}
\end{gathered}
$$

which are labelled as blue line in Fig. 3(a). Then, a high order function is used to fit these three IF curves, and the continuous IF curve function of the simulated signal is estimated as

$$
f(t)=153.8+93.2 t+11.9 t^{3}-30 t^{4}+2.8 t^{5}
$$

The DO of the simulated signal is $f_{d}(t)=\int_{0}^{t}(f(t)-153.8) d t$. The result is presented in Fig. 3 (b). Estimation error is $2.8 \%$. Hence, the proposed method is effective for nonstationary signals.
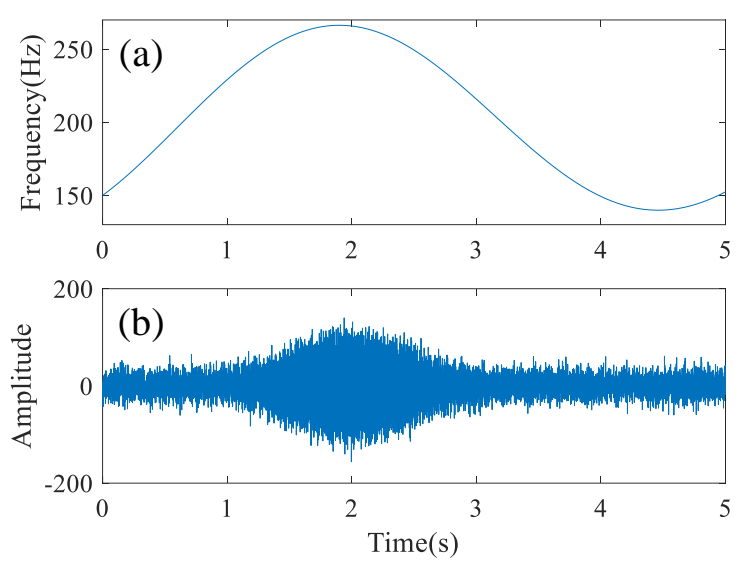

(c)

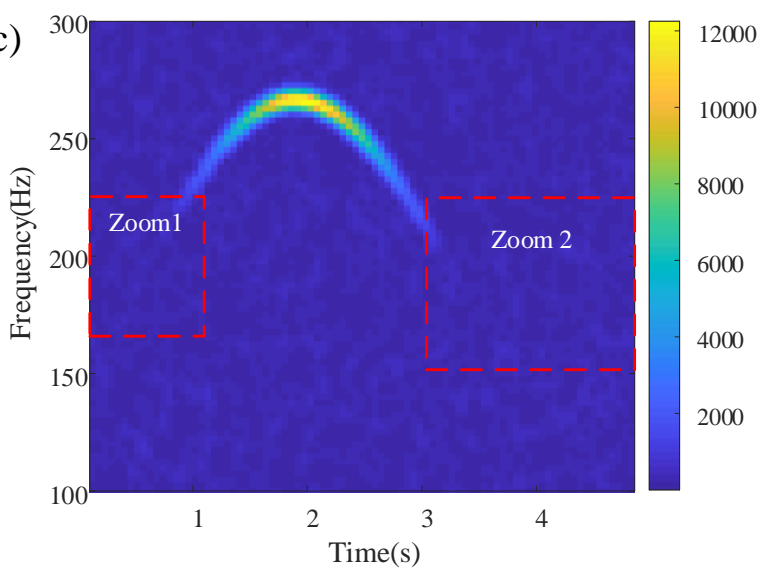

Fig.2 The simulated signal: (a) IF curve; (b) signal with noise and (c) TFR of the simulated signal
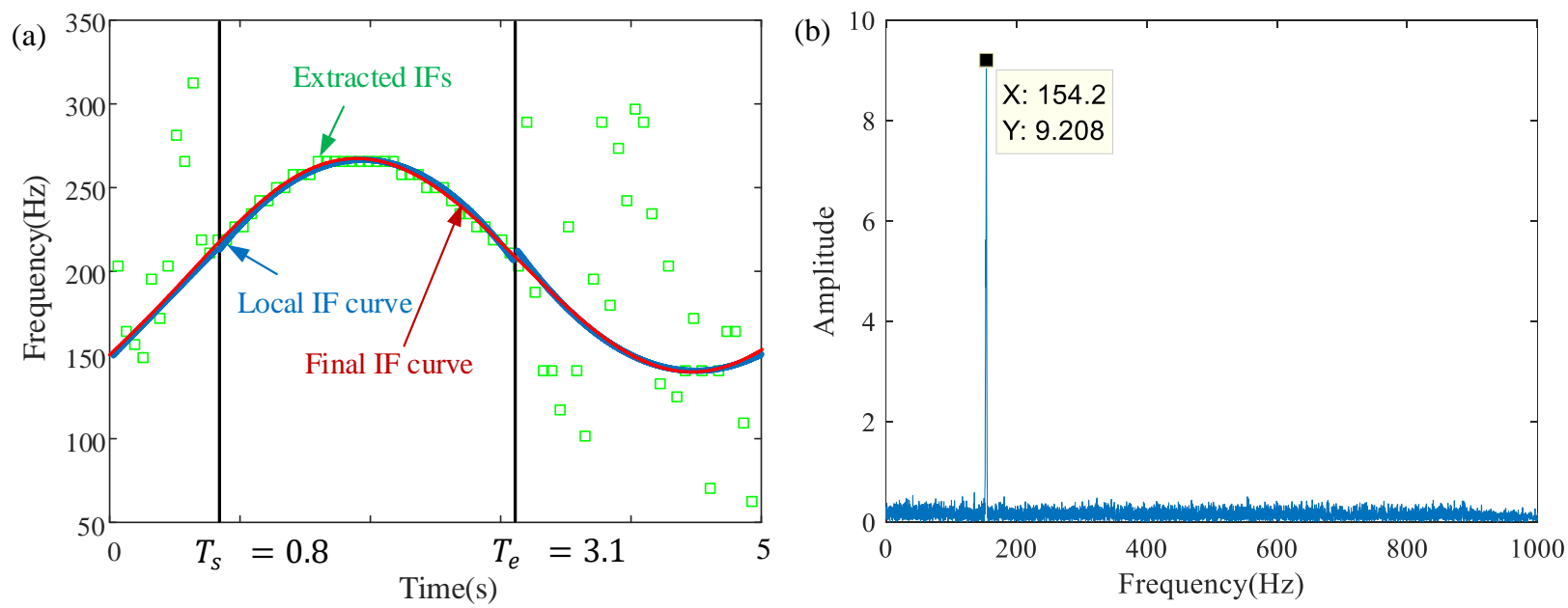

Fig.3 Demonstration results: (a) IF curve and (b) the spectrum of the demodulated signal 


\section{Rolling bearing fault detection using hypothesis based demodulation transform}

The theory and feasibility of rolling bearing fault diagnosis, based on the estimated FCFs, and hypothesis based demodulation transform are introduced in this section. The rolling bearing fault diagnosis method includes two aspects: (a) the FCFs estimation, using the proposed technology; (b) quantitatively diagnosis, using hypothesis based demodulation transform.

The harmonics of the FCF are important features for determining fault type of rolling bearings. The FCFs of outer race fault $F C F_{o}$, inner race fault $F C F_{i}$ and rolling element fault $F C F_{b}$ are estimated as [37]

$$
\begin{gathered}
F C F_{o}=F C C_{o} \cdot f_{r}=\frac{n}{2}\left(1-\frac{d}{D} \cos \alpha\right) \\
F C F_{i}=F C C_{i} \cdot f_{r}=\frac{n}{2}\left(1+\frac{d}{D} \cos \alpha\right) \\
F C F_{b}=F C C_{b} \cdot f_{r}=\frac{1}{2} \cdot \frac{d}{D}\left\{1-\left(\frac{d}{D}\right)^{2} \cos ^{2} \alpha\right\}
\end{gathered}
$$

$n$ is number of rolling elements; $f_{r}$ is RF; $d$ is diameter of rolling element; $D$ is pitch diameter; $\alpha$ is angle of load from the radial plane.

Under time-varying rotational speed conditions, the faulty bearing signal model [35] is

$$
\begin{gathered}
x_{b}(t)=\left\{1+A^{\prime}(t) \sin \left[2 \pi \int_{0}^{t} f_{r}(s) d s\right]\right\} \\
\sum_{i=1}^{I} A_{i} e^{-\beta_{b}\left(t-t_{i}-\sum_{j=1}^{i} \tau_{j}\right)} \sin \left[\omega_{b}\left(t-t_{i}-\sum_{j=1}^{i} \tau_{j}\right)\right] \cdot \mu\left(t-t_{i}-\sum_{j=1}^{i} \tau_{j}\right)
\end{gathered}
$$

where the first line describes fault-induced resonance, being demodulated by the RF $f_{r}(t)$, and the second line presents impulse series, related to local fault; $A^{\prime}(t)=\lambda_{b}^{\prime} t$ is the amplitude of the RF fundamental harmonic; $A_{i}$ is the amplitude of the $i$-th impulse, $A_{i}=\lambda_{b} t_{i} ; \beta_{b}$ represents damping characteristic; $\omega_{b}$ is frequency of resonance, excited by bearing fault; $t_{i}$ is occurrence time of the $i$-th impulse. $\tau_{j}$ is parameter, describing a random slippage of rolling elements, whose value is 0.01 and time interval between two impulses; $\mu(t)$ is the unit step function; $t_{i}$ represents occurrence time of the $i$-th impulse as

$$
t_{i}=\frac{i}{\left[F C C \times f_{r}(t)\right]}
$$

The envelope of signal $x_{b}(t)$ is,

$$
e(t)=\sqrt{x_{b}^{2}(t)+H^{2}\left[x_{b}(t)\right]}
$$

The RF and the FCFs can be identified from TFR of the envelope. The FCF curve has relatively high amplitude in TFR of envelope of faulty bearing signal, compared with the RF curve. Hence, the fault detection, based on FCF extraction and RF matching, is effective.

The main steps of hypothesis based demodulation transform can be described as (a) RF-related DOs for different fault types are estimated, based on the estimated FCFs and given FCCs; (b) corresponding demodulated signals are obtained, using the proposed demodulation transform, and the time-varying RF curve is transformed to a constant one; (c) the transformed RF is quantitatively characterized, using the spectrum, and bearing fault type is detected. 
The schematic of bearing FCF estimation and diagnosis method is shown in Fig.4 and its main steps are as

(a) Estimate the filtered signal, using spectral kurtosis-based band-pass filter.

(b) Estimate TFR of envelope of the filtered signal via the STFT.

(c) Employ the proposed transform for obtaining FCF curve.

(d) Estimate the FCFs related DOs based on the FCF curve, and corresponding spectra of the demodulated signals.

(e) Estimate three RF related DOs (related to outer race, inner race and rolling element faults), based on the FCCs and FCF curve.

(f) Estimate the demodulated signals and, corresponding spectra, based on the obtained DOs.

(g) Determine fault type by observing the spectral peak under three hypothesis: i.e., outer race fault, inner race fault and rolling element fault.

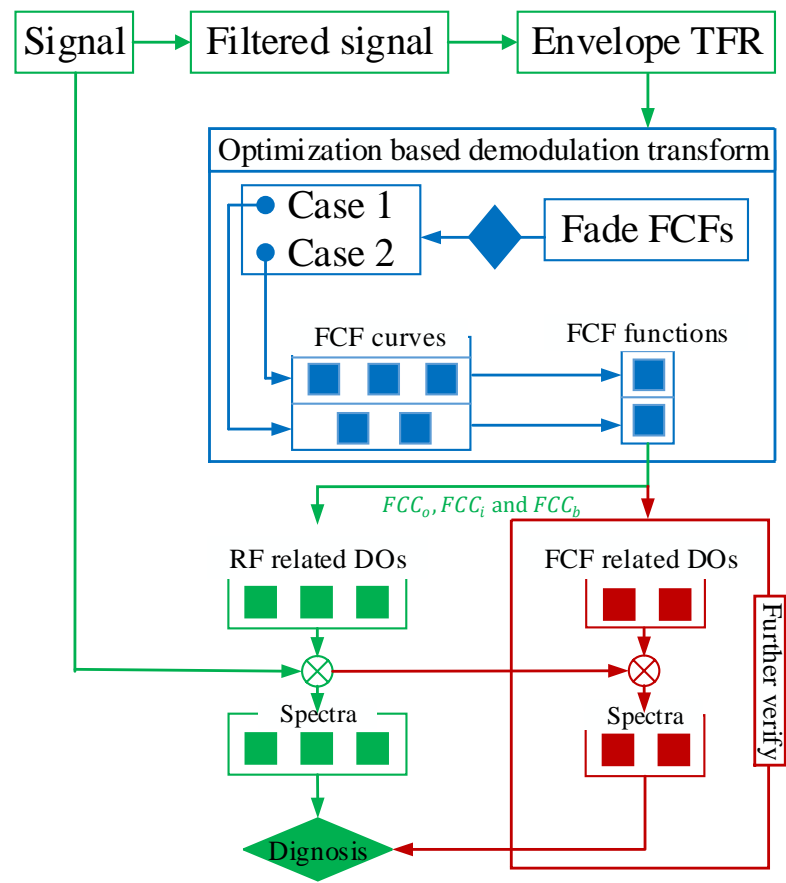

Fig.4 Schematic of bearing FCF estimation and diagnosis method

\section{Simulation analysis}

4.1 Simulation results and discussion

A simulated bearing signal with outer race fault under time-varying RF is considered for validating the effectiveness of the proposed method as

$$
x_{s}(t)=x_{b}(t)+n(t)
$$

where $n(t)$ is the Gaussian white noise. The SNR is

$$
S N R=10 \log _{10} \frac{\frac{1}{N} \sum_{n=0}^{N-1}\left|x_{b}(t)\right|^{2}}{\frac{1}{N} \sum_{n=0}^{N-1}|n(t)|^{2}}
$$

The parameters of the simulation model are listed in Table 1.

Table 1 Parameters of the simulated model 


\begin{tabular}{ll}
\hline Parameter & Value \\
\hline Sampling rate, $f_{s} / \mathrm{Hz}$ & 20000 \\
FCC & $F C C_{o}=3.5, F C C_{i}=4.6$ and $F C C_{b}=2.8$ \\
Damping characteristic, $\beta_{b}$ & 800 \\
Resonance frequency of bearing, $\omega_{b} / \mathrm{Hz}$ & 5000 \\
RF, $f_{r} / \mathrm{Hz}$ & $f_{r}(t)=-t^{2}+12.6 t+19$ \\
Amplitude coefficient, $\lambda_{b}^{\prime}, \lambda_{b}$ & $\lambda_{b}^{\prime}=0.2 ; \lambda_{b}=0.3$ \\
SNR, dB & -1 \\
\hline
\end{tabular}

The bearing impulse series are shown in Fig.5 (a), via red lines and the simulated signal is plotted by blue line in Fig.5 (a). The envelope, (Fig.5 (b)), is extracted from the simulated signal, using the spectral kurtosis-based filter (filtering band is $3700 \mathrm{~Hz}$ to $6300 \mathrm{~Hz}$ ) and the Hilbert transform. The TFR of the envelope (Fig.5 (c)), shows, that fault related time-frequency ridges (the RF, the FCF and its multiples) are buried by noise, and only a part of the FCF is clearly seen in high RF region. The FCFs (Fig.5 (d), red squares) are extracted from the TFR.

During $0-2 \mathrm{~s}$, the FCFs cannot be extracted accurately. Error of the fitted FCF curve (green line in Fig.5 (d)) is $30 \%$. Case 1 is selected for determining the local model, and $T_{e}=2$, i.e. $0 \leq I_{1}<2$ and $2 \leq$ $I_{2} \leq 5$. After the processing procedures, described in Section 2.3, the FCF curve in the first section is estimated as $\left.f_{F C F}(t)\right|_{t \in I_{1}}=68.6+38.8 t-2.1 t^{2}$. The FCF curve in the second section is estimated by fitting the extracted FCFs $\left.f_{F C F}(t)\right|_{t \in I_{2}}=70.7+39.8 t-2.9 t^{2}$. The final FCF curve is estimated as $f_{F C F}(t)=67.4+42.6 t-3.3 t^{2}$, whose error is $1.4 \%$ (Table 2). The FCF curve, obtained by the peak searching algorithm, is also presented in Table 2, and error is $48.2 \%$. By comparison, the proposed method has better ability for FCF estimation.

Three RF related DOs are estimated based on the FCCs and FCF function. They are listed in Table 2. The demodulated signal is obtained, using the DOs and corresponding spectra are estimated (Fig. 5 (e)). From the spectra, the outer race fault related peak at frequency $(f=18.86)$ is only found and error is $0.5 \%$. The FCF and its second multiple are estimated and corresponding peaks at frequencies $(f=66.3)$ and $(f=132.8)$ are also be captured (Fig. 5(f)). Errors of the FCF and multiples are $0.3 \%$ and $0.2 \%$, respectively. As a result, the bearing fault is determined, using the proposed method, with a satisfactory accuracy.

Table 2 FCF functions and Dos for the proposed method and the GDT

\begin{tabular}{lllll}
\hline & \multicolumn{2}{c}{ The proposed method } & \multicolumn{2}{c}{ The traditional GDT } \\
\cline { 2 - 5 } & FCF function & Error & FCF function & Error \\
\cline { 2 - 5 } & $f_{F C F}(t)=67.4+42.6 t-3.3 t^{2}$ & $1.4 \%$ & $f_{F C F 2}(t)=97.1+26.3 t-0.8 t^{2}$ & $48.2 \%$ \\
\hline DO: Outer race & $s_{o}(t)=\int_{0}^{t} f_{F C F}(t)-67.4 d t / F C C_{o}$ & $s_{o 2}(t)=\int_{0}^{t} f_{F C F 2}(t)-97.1 d t / F C C_{o}$ \\
DO: Inner race & $s_{i}(t)=\int_{0}^{t} f_{F C F}(t)-67.4 d t / F C C_{i}$ & $s_{i 2}(t)=\int_{0}^{t} f_{F C F 2}(t)-97.1 d t / F C C_{i}$ \\
DO: Ball & $s_{b}(t)=\int_{0}^{t} f_{F C F}(t)-67.4 d t / F C C_{b}$ & $s_{b 2}(t)=\int_{0}^{t} f_{F C F 2}(t)-97.1 d t / F C C_{b}$ \\
\hline
\end{tabular}



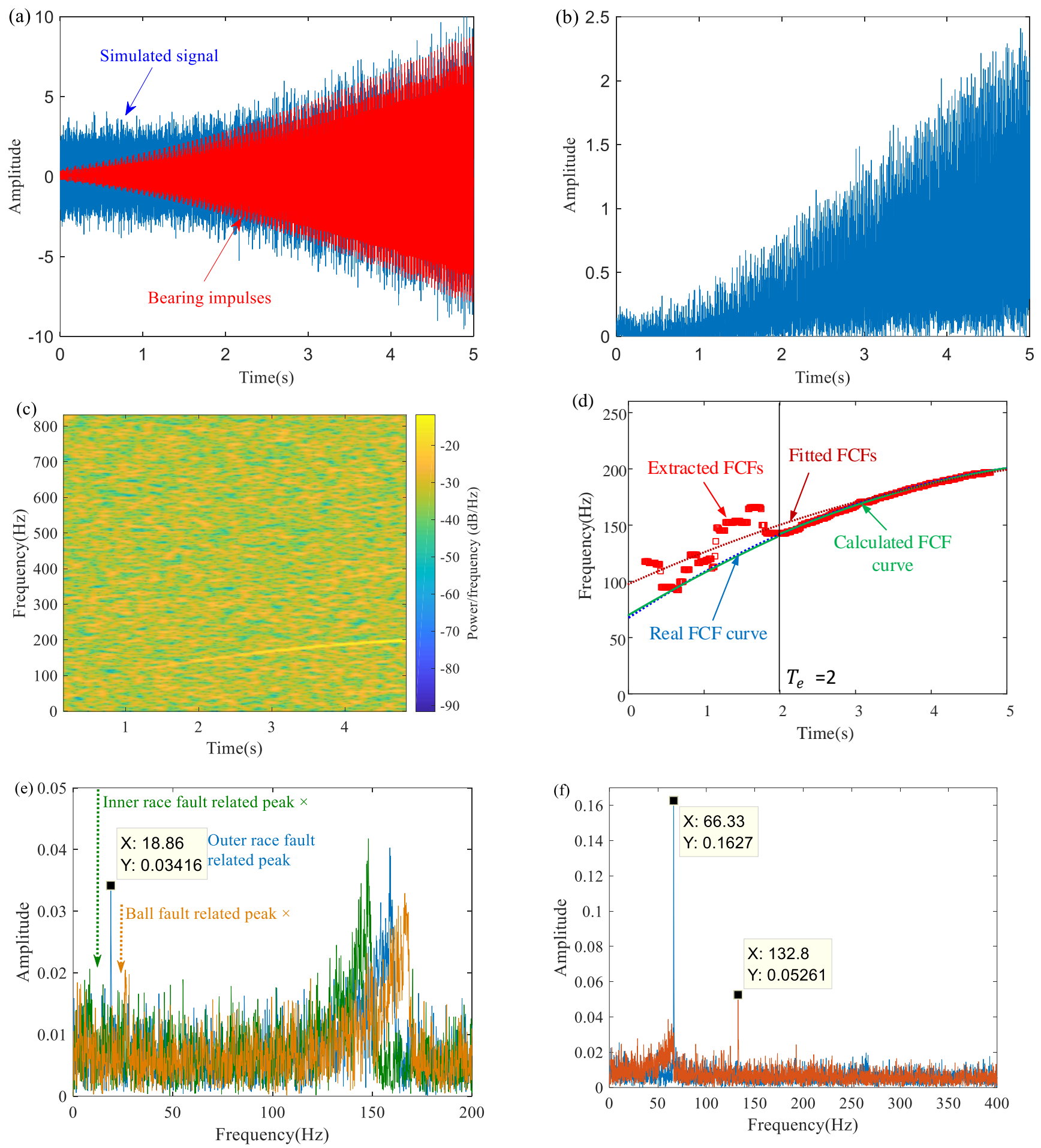

Fig.5 (a) The simulated signal; (b) envelope signal of the filtered signal; (c) the STFT; (d) the extracted FCFs; (e) RF-related spectra; (f) FCF-related spectra.

\subsection{Comparison analysis}

For novel comparison, results, estimated by the traditional GDT, are plotted in Fig. 6 (a) and (b). The RF related DOs are estimated, based on the FCF function $f_{F C F 2}(t)$ (Table 2) and the FCCs. From Fig. 6(a), the outer race fault related peak at frequency $(f=28.3)$ is found. However, error is $48.9 \%$. In addition, spectral smearing is found in the FCF-related spectra, especially for the harmonic, shown in Fig. 6 (b). The results, obtained by the GDT, have a higher estimation error, are influenced by noise, and, therefore, cannot be effectively used to detect bearing fault. 
The results, related to the proposed method and the GDT, are listed in Table 3. It can be seen, that the proposed method is more effective, than the traditional method, for detecting bearing fault under timevarying speed conditions.
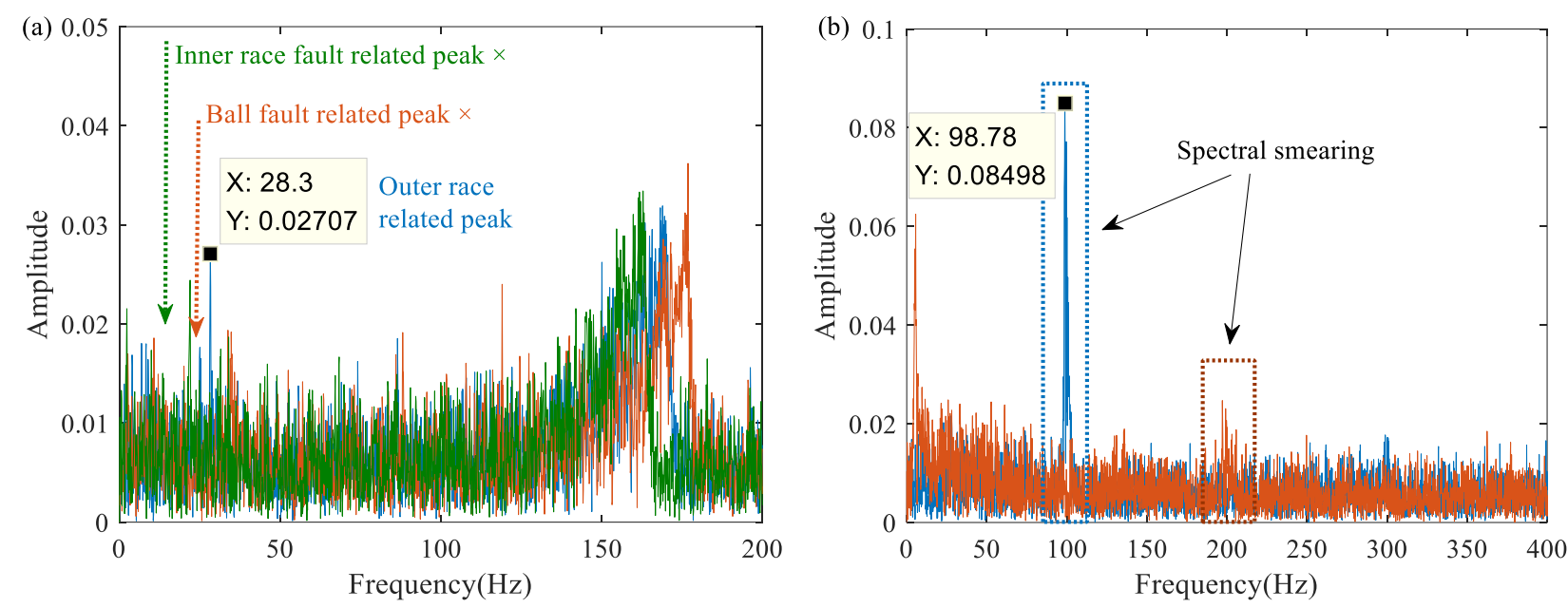

Fig.6 Traditional GDT results, related to the simulated signal: (a) RF-related spectra; (b) FCF-related spectra

Table 3 Comparison results, related to the simulated signal

\begin{tabular}{lllllll}
\hline \multirow{2}{*}{ Fault type } & \multirow{2}{*}{$\begin{array}{l}\text { Fault } \\
\text { feature }\end{array}$} & \multirow{2}{*}{$\begin{array}{l}\text { Theory } \\
\text { value }\end{array}$} & \multicolumn{2}{c}{ Proposed method } & \multicolumn{2}{c}{ Traditional GDT } \\
\cline { 5 - 7 } & Estimation & error & Estimation & error \\
\hline Outer race & RF & 19 & 18.9 & $0.5 \%$ & 28.3 & $48.9 \%$ \\
& FCF & 66.5 & 66.3 & $0.3 \%$ & 98.8 & $48.6 \%$ \\
& $2 \times$ FCF & 133 & 132.8 & $0.2 \%$ & $\times$ & $\times$ \\
\hline
\end{tabular}

\section{Experimental validation}

\subsection{Experimental result discussion}

The bearing vibration signal, measured from the SpectraQuest machinery fault simulator, (Fig. 7), is used to evaluate diagnosis performances of the proposed method. The experimental setup consists of a motor, whose rotational speed is controlled by an AC converter, and a built-in tachometer, that is used to measure rotational speed. The shaft is supported by two rolling bearings, including a faulty one (left), that has an outer race local fault. A defect in form of $0.1 \mathrm{~mm}$ diameter hole is considered in the outer raceway of the bearing; the relative damage size, related to the outer race circumference, is $0.2 \%$. Two flywheels are installed on the shaft to apply load to the system. The vibration signal is collected by an accelerometer PCB 352A60, frequency response from $5 \mathrm{~Hz}$ to $60 \mathrm{kHz}$, the sensitivity is $10 \mathrm{mV} / \mathrm{g}$. It is mounted on the top surface of the faulty bearing housing. Parameters of faulty bearing are listed in Table 4. 


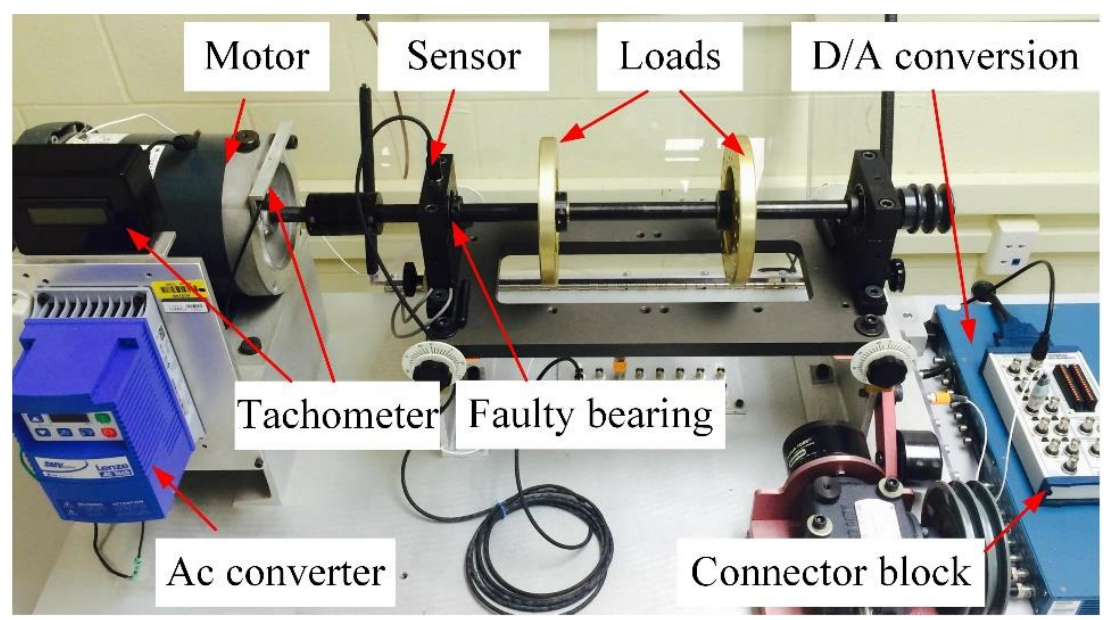

Fig. 7 Experimental setup for bearing fault diagnosis

Table 4 Parameters of faulty bearing

\begin{tabular}{ll}
\hline Parameter & Value \\
\hline Bearing type & ER-10k \\
Rolling element diameter $d / \mathrm{mm}$ & 7.94 \\
Pitch diameter $D / \mathrm{mm}$ & 33.5 \\
Number of rolling elements $n_{b}$ & 8 \\
Contact angle $\alpha$ & 0 \\
FCC of the outer race $F C C_{o}$ & 3.052 \\
FCC of the inner race $F C C_{i}$ & 4.95 \\
FCC of the ball $F C C_{b}$ & 1.99 \\
\hline
\end{tabular}

The RF of the shaft gradually increases from $16.7 \mathrm{~Hz}$ to $31.2 \mathrm{~Hz}$ and then goes down to $10 \mathrm{~Hz}$. The signal duration is $10 \mathrm{~s}$ and the sampling frequency is $48000 \mathrm{~Hz}$. The measured vibration signal is shown in Fig. 8 (a). Fig. 8 (b) is envelope signal of the signal, filtered by the spectral kurtosis based band-pass filter, frequency band is from $8000 \mathrm{~Hz}$ to $9500 \mathrm{~Hz}$. The STFT is employed to the envelope signal. From the TFR (Fig. 8(c)), the visible time-frequency ridges are found only in a middle local region, and the time-frequency ridges in starting and stopping regions are completely overwhelmed by a noise. This phenomenon is also testified by the extracted FCFs (Fig. 8(d)).

The proposed method is applied to the envelope signal. The case 2 is selected. The starting point $T_{S}$ and the ending point $T_{e}$ are set to 1.5 and 8.5 respectively. Namely, $I_{1}=[0,1.5], I_{2}=[1.5,8.5]$ and $I_{3}=$ $[8.5,10]$. The FCF functions $\left(\left.f_{F C F}(t)\right|_{t \in I_{1}},\left.f_{F C F}(t)\right|_{t \in I_{2}}\right.$ and $\left.f_{F C F}(t)\right|_{t \in I_{3}}$ ) for all local models are shown in Table 5. Hence, the integral function of FCF $f_{F C F_{-} c}(t)$ is estimated (Table 5), and error is $4 \%$. The actual FCF function, calculated by the RF function and $\mathrm{FCO}_{o}$ is also presented in Table 5. In addition, the FCF function $f_{F C F_{-} t r a}(t)$, estimated by fitting the extracted FCFs, is presented in Table 5, and its error is $38 \%$.

Based on the estimated FCFs, the RF-related DOs for fault hypotheses are listed in Table 6. The envelope signal is demodulated by the DOs $s_{o}(t), s_{i}(t)$ and $s_{b}(t)$, respectively. The corresponding spectra are in Fig. 8(e), using different colours, i.e., blue is related to outer race spectrum, green is related to inner race spectrum, orange is related to rolling element spectrum. From Fig. 8 (e), only outer race fault related peak can be clearly seen and error is 3.6\%. The FCF harmonic related spectra are shown in Fig. 8 (f), from which the corresponding peaks are also clearly seen. The error of FCF peak is $2.4 \%$ and the error of second harmonic is $2.8 \%$. 
Hence, the proposed method is effective to diagnose bearing fault.

Table 5 FCF functions for the proposed method and the GDT

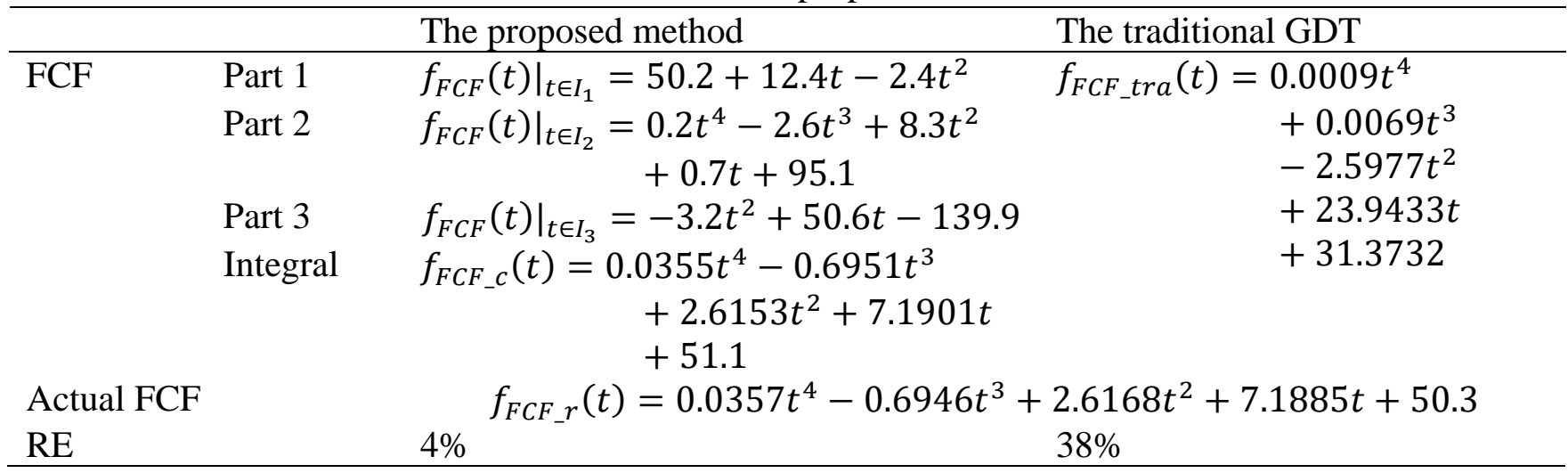

Table 6 DOs

\begin{tabular}{lll}
\hline & \multicolumn{1}{c}{ Proposed method } & \multicolumn{1}{c}{ Traditional GDT } \\
\hline DO: Outer race & $s_{o}(t)=\int_{0}^{t} f_{F C F_{-} c}(t)-67.4 d t / F C C_{o}$ & $s_{o 2}(t)=\int_{0}^{t} f_{F C F_{-} t r a}(t)-97.1 d t / F C C_{o}$ \\
DO: Inner race & $s_{i}(t)=\int_{0}^{t} f_{F C F_{-} c}(t)-67.4 d t / F C C_{i}$ & $s_{i 2}(t)=\int_{0}^{t} f_{F C F_{-} t r a}(t)-97.1 d t / F C C_{i}$ \\
DO: Ball & $s_{b}(t)=\int_{0}^{t} f_{F C F_{-} c}(t)-67.4 d t / F C C_{b}$ & $s_{b 2}(t)=\int_{0}^{t} f_{F C F_{-} \text {tra }}(t)-97.1 d t / F C C_{b}$ \\
\hline
\end{tabular}
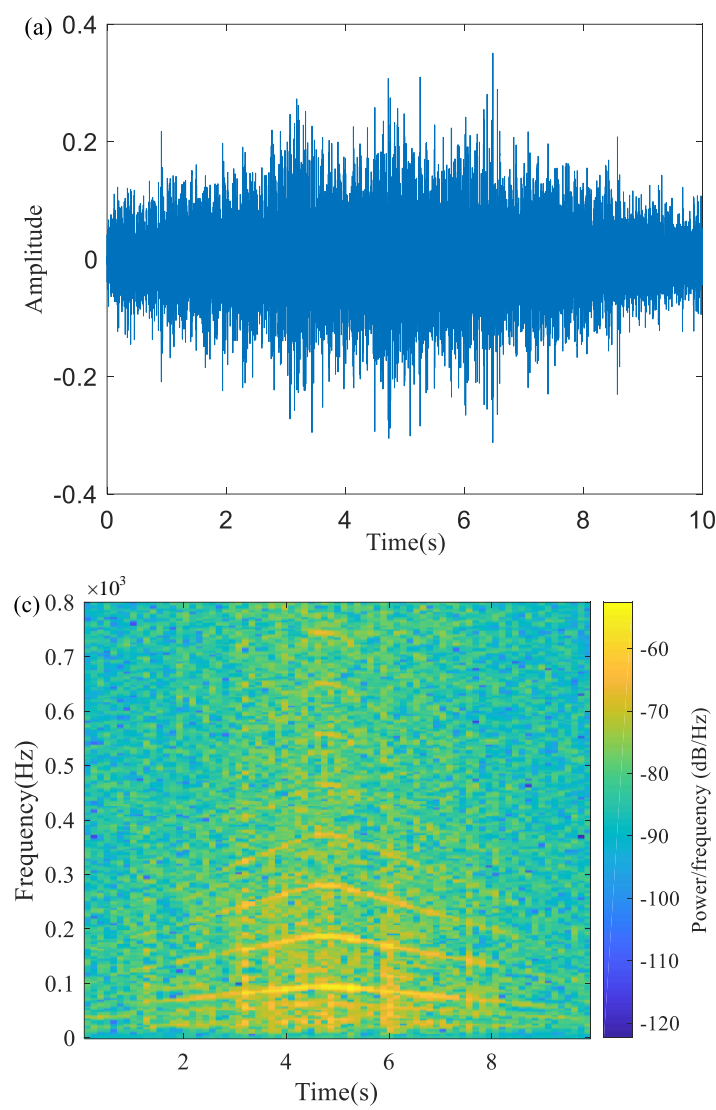
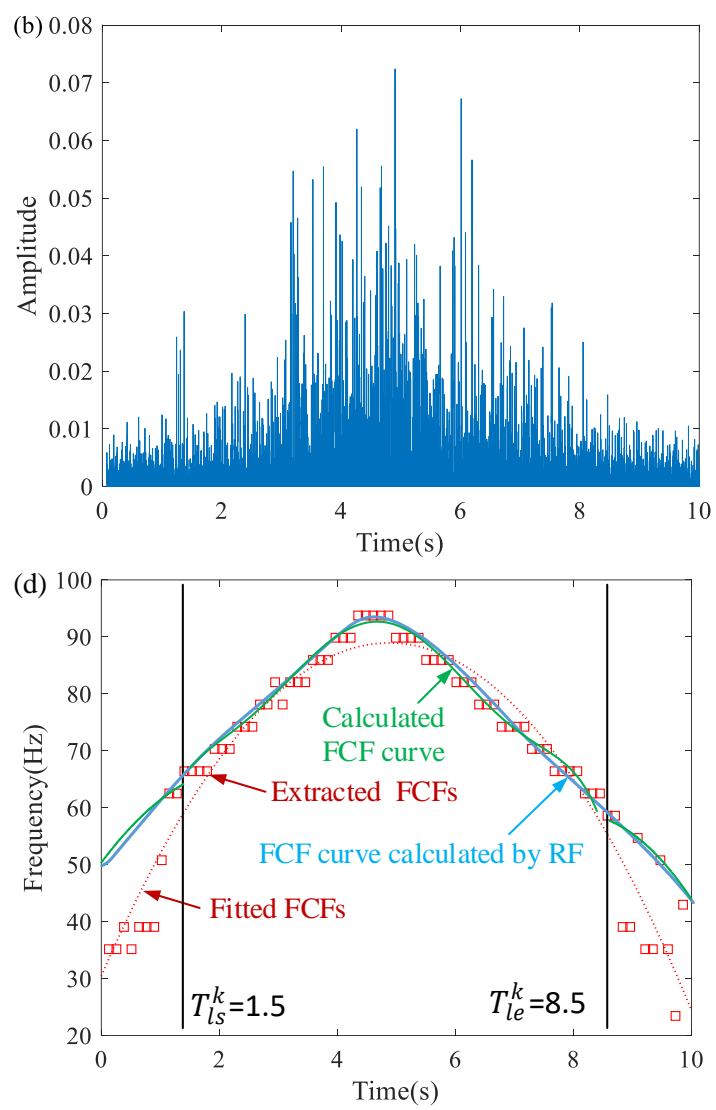

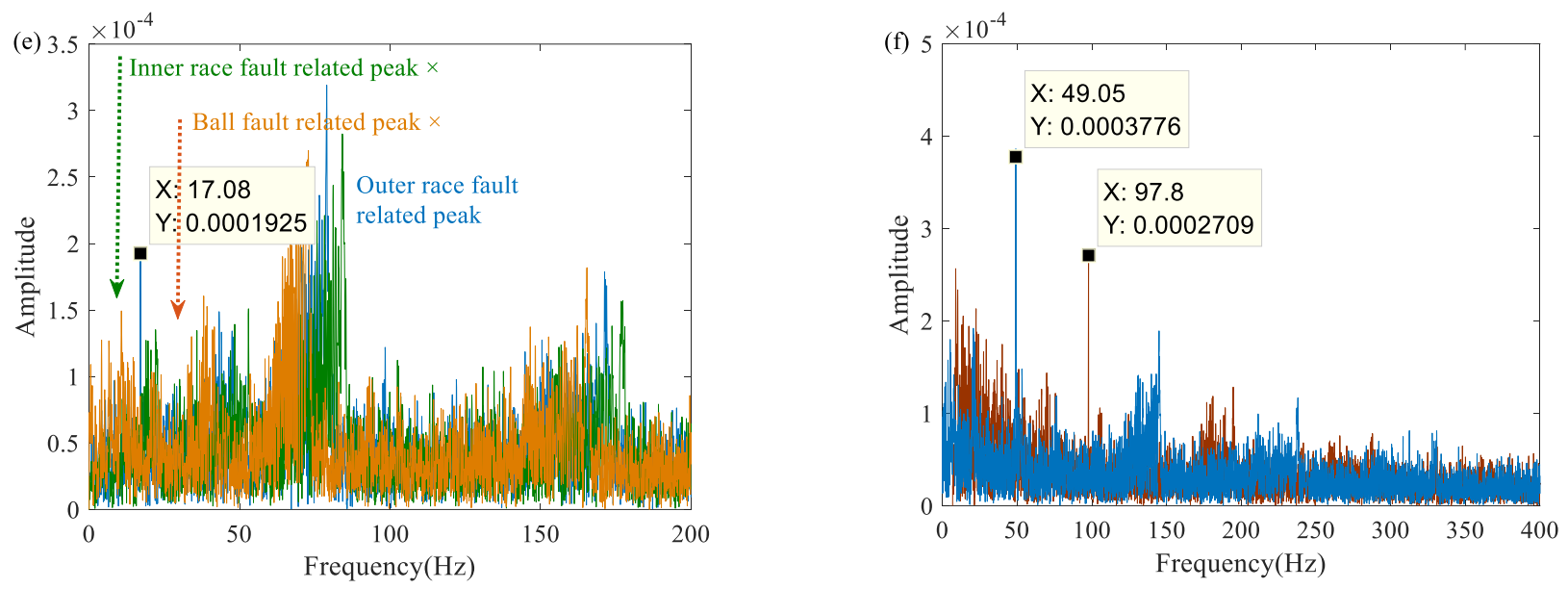

Fig.8 Analysis results of experimental signal: (a) the measured signal; (b) envelope signal of filtered signal; (c) TFR; (d) extracted FCFs; (e) RF-related spectra; (f) FCF-related spectra.

\subsection{Comparison analysis}

Five novel comparisons are discussed to further demonstrate the effectiveness of the proposed bearing fault diagnosis method.

Firstly, novel comparison is made between the proposed technique and the wavelet transform (WT) [39], the SST [22] and the synchroextracting transform (SET) [23]. The TFRs of envelope signal, obtained by these three methods, are shown in Fig.9 (a), (b) and (c), respectively. The peak searching method is applied to extract the FCFs from the TFRs, and the extracted FCFs and actual FCFs are presented in Fig.9 (d). The searching parameters for traditional methods are the same as for proposed method. From Fig. 9 (d), it can be found, that the FCFs cannot be accurately extracted by the considered TFA methods, and the problem, related to the time-varying impulse amplitude, cannot be addressed.

Secondly, novel comparison is made with method [37], The partial FCFs are selected from the extracted FCFs (Fig.9(d)) for bearing fault diagnosis by method [37]. The selected FCFs are not actual and some information is missing. Hence, the proposed method is more effective for diagnosis, than method [37].

Thirdly, novel comparison is made between the traditional GDT and the proposed technique. For the GDT bearing fault diagnosis method, the FCF-related DOs are estimated, based on the estimated FCFs from TFR (Table 6). The envelope signal is demodulated by the DOs $s_{o 2}(t), s_{i 2}(t)$ and $s_{b 2}(t)$, respectively. The corresponding spectra are in Fig. 10 (a) using different colours, i.e., blue is related to outer race spectrum, green is related to inner race spectrum, orange is related to rolling element spectrum. There is no fault related peak in Fig. 10 (a). In addition, there is spectral smearing phenomenon in the FCF and its multiples in Fig. 10 (b). Hence, the bearing fault cannot be detected by the GDT. Results are in Table 7. It shows, that the proposed method has better ability to diagnose bearing under time-varying rotational speeds. 
(a)

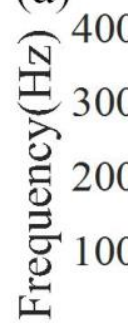

(c)

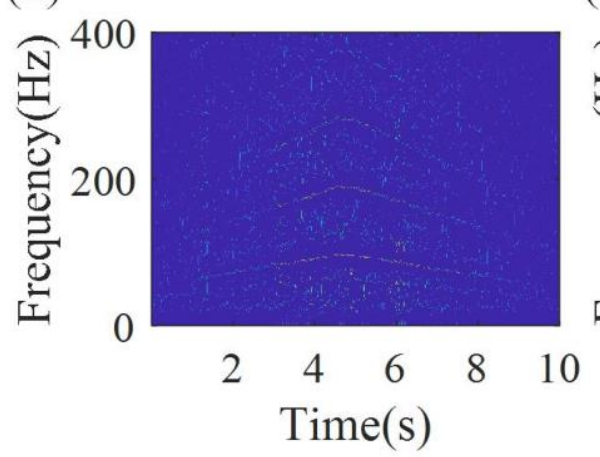

(b)

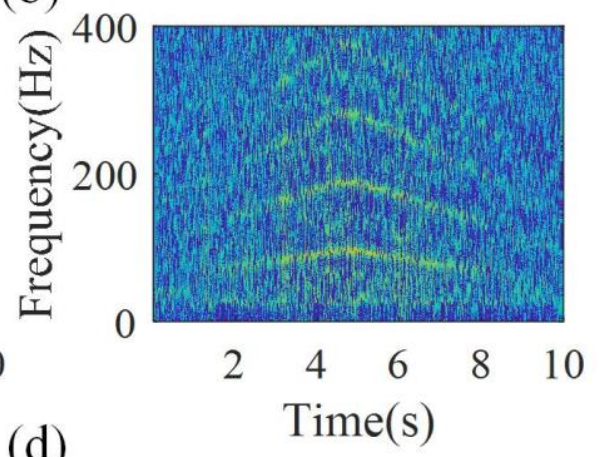

(d)

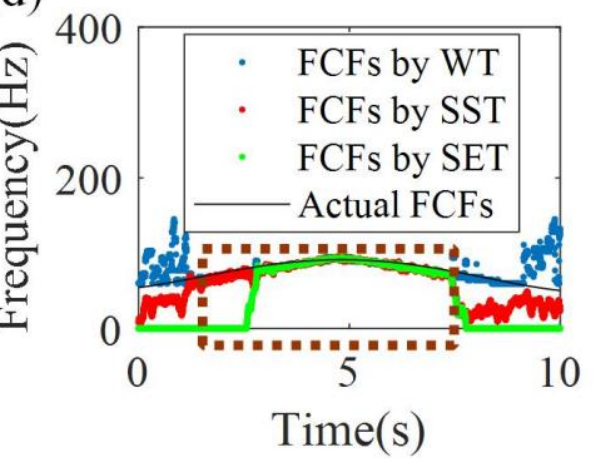

Fig.9 Comparison results with advanced TFR methods: TFRs obtained by (a) the WT, (b) the SST and (c) the SET; (d) the extracted FCFs from above three TFRs
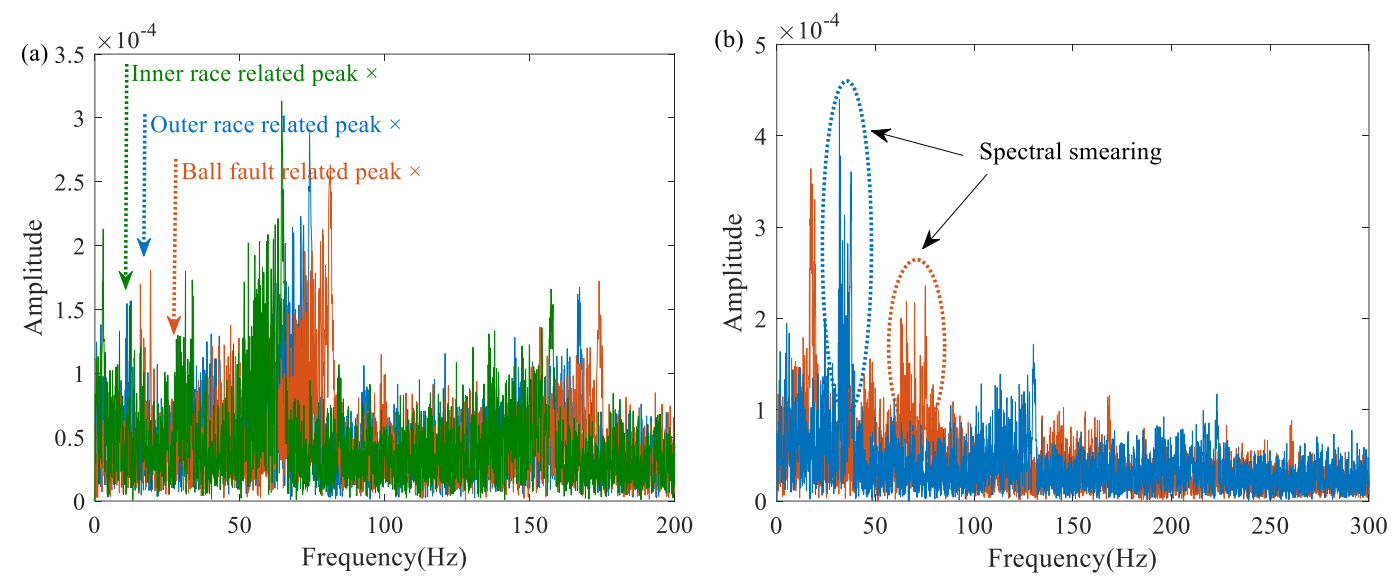

Fig.10 Traditional GDT results of the measured signal: (a) RF-related spectra; (b) FCF-related spectra

Table 7 Comparison results for the measured signal

\begin{tabular}{lllllll}
\hline \multirow{2}{*}{ Fault type } & \multirow{2}{*}{$\begin{array}{l}\text { Fault } \\
\text { feature }\end{array}$} & Theory & \multicolumn{2}{c}{ The proposed method } & \multicolumn{2}{c}{ The traditional GDT } \\
\cline { 4 - 7 } & RF & 16.5 & Estimation & error & Estimation & error \\
\hline Outer race & RF & 17.1 & $3.6 \%$ & $\times$ & $\times$ \\
& FCF & 50.3 & 49.1 & $2.4 \%$ & $\times$ & $\times$ \\
& $2 \times \mathrm{FCF}$ & 100.6 & 97.8 & $2.8 \%$ & $\times$ & $\times$ \\
\hline
\end{tabular}

As it is not known a priori, regarding bearing fault existence/non-existence, for cases of non-existence of bearing faults in noisy environment, the method will make the same steps, as for cases of fault existence, as follows:

- Apply the Hilbert transform to the bearing vibration signals for obtaining an envelope signal. 
- Obtain a TFR of envelope signal by the SFTF.

- Obtain IFs, which are essentially polluted by noise, from a TFR, by the peak searching algorithm.

- Estimate IF curve parameters for the selected intervals, using the optimization algorithm.

- Estimate IF curve, i.e. RF.

- Estimate the DO, related to the outer race fault frequency, based on the estimated RF and the outer race $\mathrm{FCC}$.

- Demodulate the analytic signal, using the estimated DO.

- Detect peaks in the spectrum of the demodulated signal. As in non-faulty cases peaks will not be detected, the following result will be obtained: a decision, that "no outer race fault".

- Estimate the DO, related to the inner race fault frequency, based on the estimated RF and the inner race FCC.

- Demodulate the analytic signal, using the estimated DO.

- Detect peaks in the spectrum of the demodulated signal. As in non-faulty cases peaks will not be detected, the following result will be obtained: a decision, that "no inner race fault".

- Estimate the DO, related to the rolling element fault frequency, based on the estimated RF and the rolling element FCC.

- Demodulate the analytic signal, using the estimated DO.

- Detect peaks in the spectrum of the demodulated signal. As in non-faulty cases peaks will not be detected, the following result will be obtained: a decision, that "no rolling element fault".

\section{Conclusions}

The novel IF estimation method for rolling bearings and novel bearing diagnosis method under variable speeds are developed and validated by the simulated and the experimental data for conditions of timevarying impulse amplitudes and time-varying impulse intervals, related to bearing faults. The proposed technology is a novel generalisation of the GDT in non-stationary conditions of variable impulse amplitudes, related to bearing faults.

(a) The results, related to the simulated and the experimental vibration data, show, that the proposed method can effectively estimate the bearing FCF, with low errors $0.3 \%$ and $2.4 \%$, respectively, and detect bearing fault at early stage of fault development $(0.2 \%$ relative fault size $)$ under time-varying rotational speeds and the errors of the RF estimation are $0.5 \%$ and $3.6 \%$, respectively. Weak FCF under low speed is accurately estimated, using the optimization based demodulation transform, and the challenge, related to time-varying impulse amplitude, is overcome. The time-varying RF is transformed into a constant line, using the hypothesis based demodulation transform, and then it is quantitatively characterized for bearing fault diagnosis. The novel hypothesis based parameter optimization of the proposed technology is successfully validated.

(b) Five novel experimental comparisons in essentially noisy environment show that a) comparing with the three advanced TFA based bearing fault diagnosis methods, the proposed technique is more effective for estimation of weak FCF with low error, 2.4\%; in contrast, the FCF cannot be effectively estimated, using the advanced TFA and peak searching algorithm; b) comparing with the traditional 
GDT-based bearing fault diagnosis methods, the proposed technique is more effective for estimation of weak FCF and fault diagnosis.

The proposed method could be extended for other rotating machinery and structures (e.g. gearboxes [39], engines [41], civil engineering structures [42], etc.) and make an influence on vibration based health monitoring of rotating machinery and structures under nonstationary conditions.

\section{Acknowledgments}

This study is supported by the National Natural Science Foundation of China (Grant No. 51905292) and China Postdoctoral Science Foundation (2019M660615 and 2020T130348).

\section{References}

[1] Ciszewski T, Gelman L, Swędrowski L. Current-based higher-order spectral covariance as a bearing diagnostic feature for induction motors. Insight: Non-Destructive Testing and Condition Monitoring, 58(2016), 431-434.

[2] Freire W M, Lucchesi CK. Experimental validation and comparison between different active control methods applied to a journal bearing supported rotor. Structural Control and Health Monitoring 26.12 (2019): e2446.

[3] Gelman L, Murray B, Patel T H, Thomson A. Novel wavelet technology for vibration condition monitoring of rolling element bearings. Insight: Non-Destructive Testing and Condition Monitoring, 57(1)(2015), 40-47.

[4] Wu G, Yang D, Yi T, Li H, Liu H. Sliding life prediction of sliding bearings using dynamic monitoring data of bridges. Structural Control and Health Monitoring 27.5 (2020): e2515.

[5] Ciszewski T, Gelman L, Ball A. Novel fault identification for electromechanical systems via spectral technique and electrical data processing, Electronics, 9(2020), 1-20.

[6] Zhao D, Li J, Cheng W, He Z. Generalized demodulation transform for bearing fault diagnosis under nonstationary conditions and gear noise interferences. Chinese Journal of Mechanical Engineering 32.1 (2019): 7.

[7] Loh CH, Loh KJ, Yang YS, Hsiung WY, Huang, YT. Vibration-based system identification of wind turbine system. Structural Control and Health Monitoring 24.3 (2017): e1876.

[8] Yuan H, Lu C. Rolling bearing fault diagnosis under fluctuant conditions based on compressed sensing. Structural Control and Health Monitoring 24.5 (2017): e1918.

[9] Zhao D, Cheng W, Gao RX, Yan R, Wang P. Generalized Vold-Kalman filtering for nonstationary compound faults feature extraction of bearing and gear. IEEE Transactions on Instrumentation and Measurement (2019) 69(2), 401-410.

[10] Gelman L, Patel TH, Persin G, Murray B, Thomson A. Novel technology based on the spectral kurtosis and wavelet transform for rolling bearing diagnosis. International Journal of Prognostics and Health Management, (2013): 2153-2648.

[11] Randall RB, Antoni J. Rolling element bearing diagnostics-A tutorial. Mechanical systems and signal processing 25.2 (2011): 485-520. 
[12] Gelman L, Patel TH. Novel rolling bearing diagnosis technology using spectral kurtosis and the wavelet higher-order spectra. Insight: Non-Destructive Testing and Condition Monitoring, 57(2015), 452-456.

[13] Wang T, Liang M, Li J, Cheng W. Rolling element bearing fault diagnosis via fault characteristic order (FCO) analysis. Mechanical Systems and Signal Processing 45.1 (2014): 139-153.

[14] Kang M, Kim J, Wills LM, Kim JM. Time-varying and multiresolution envelope analysis and discriminative feature analysis for bearing fault diagnosis. IEEE Transactions on Industrial Electronics 62.12 (2015): 7749-7761.

[15] Zhao D, Gelman L, Chu F, Ball A. Novel method for vibration sensor-based instantaneous defect frequency estimation for rolling bearings under non-stationary conditions. Sensors, 20(2020), 5201.

[16] Gelman L, Murray B, Patel TH, Thomson A. Vibration diagnostics of rolling bearings by novel nonlinear non-stationary wavelet bicoherence technology. Engineering structures 80 (2014), 514-520.

[17] Liu J, Shao Y, Zuo M. The effects of the shape of localized defect in ball bearings on the vibration waveform. Proceedings of the Institution of Mechanical Engineers, Part K: Journal of Multi-body Dynamics 227.3 (2013): 261-274.

[18] Bossley KM, Mckendrick RJ, Harris CJ, Mercer C. Hybrid computed order tracking. Mechanical systems and signal processing 13.4 (1999): 627-641.

[19] Borghesani P, Ricci R, Chatterton S, Pennacchi P. A new procedure for using envelope analysis for rolling element bearing diagnostics in variable operating conditions. Mechanical Systems and Signal Processing 38.1 (2013): 23-35.

[20] Liu D, Cheng W, Wen W. Generalized demodulation with tunable E-Factor for rolling bearing diagnosis under time-varying rotational speed. Journal of Sound and Vibration 430 (2018): 59-74.

[21] Feng Z, Chen X. Adaptive iterative generalized demodulation for nonstationary complex signal analysis: Principle and application in rotating machinery fault diagnosis. Mechanical Systems and Signal Processing 110 (2018): 1-27.

[22] Auger F, Flandrin P, Lin YT, McLaughlin S, Meignen S, Oberlin T, Wu HT. Time-frequency reassignment and synchrosqueezing: An overview. IEEE Signal Processing Magazine 30.6 (2013): $32-41$.

[23] Yu G, Yu M, Xu, C. Synchroextracting transform. IEEE Transactions on Industrial Electronics 64.10 (2017): 8042-8054.

[24] Guo Y, Liu TW, Na J, Fung RF. Envelope order tracking for fault detection in rolling element bearings. Journal of Sound and Vibration 331.25 (2012): 5644-5654.

[25] Wang Y, Peter WT, Tang B, Qin Y, Deng L, Huang T, Xu G. Order spectrogram visualization for rolling bearing fault detection under speed variation conditions. Mechanical Systems and Signal Processing 122 (2019): 580-596.

[26] Saavedra PN, Rodriguez CG. Accurate assessment of computed order tracking. Shock and Vibration 13.1 (2006): 13-32.

[27] Cheng W, Gao RX, Wang J, Wang T, Wen W, Li J. Envelope deformation in computed order tracking and error in order analysis. Mechanical Systems and Signal Processing 48.1-2 (2014): 92-102. 
[28] Li C, Sanchez V, Zurita G, Lozada MC, Cabrera D. Rolling element bearing defect detection using the generalized synchrosqueezing transform guided by time-frequency ridge enhancement. ISA transactions 60 (2016): 274-284.

[29] Yu G. A concentrated time-frequency analysis tool for bearing fault diagnosis. IEEE Transactions on Instrumentation and Measurement (2019).

[30] Shi J, Liang M, Necsulescu DS, Guan Y. Generalized stepwise demodulation transform and synchrosqueezing for time-frequency analysis and bearing fault diagnosis. Journal of Sound and Vibration 368 (2016): 202-222.

[31] Olhede S, Andrew T W. A generalized demodulation approach to time-frequency projections for multicomponent signals. Proceedings of the Royal Society A: Mathematical, Physical and Engineering Sciences 461.2059 (2005): 2159-2179.

[32] Yang Y, Peng Z, Dong X, Zhang W, Meng G. General parameterized time-frequency transform. IEEE Transactions on Signal Processing 62.11 (2014): 2751-2764.

[33] Zhao D, Li J, Cheng W, Wen W. Compound faults detection of rolling element bearing based on the generalized demodulation algorithm under time-varying rotational speed. Journal of Sound and Vibration 378 (2016): 109-123.

[34] Huang W, Gao G, Li N, Jiang X, Zhu Z. Time-frequency squeezing and generalized demodulation combined for variable speed bearing fault diagnosis. IEEE Transactions on Instrumentation and Measurement 68.8 (2018): 2819-2829.

[35] Shi J, Liang M, Guan Y. Bearing fault diagnosis under variable rotational speed via the joint application of windowed fractal dimension transform and generalized demodulation: a method free from prefiltering and resampling. Mechanical Systems and Signal Processing 68 (2016): 15-33.

[36] Liu D, Cheng W, Wen W. An online bearing fault diagnosis technique via improved demodulation spectrum analysis under variable speed conditions. IEEE Systems Journal (2019).

[37] Zhao D, Wang T, Gao RX, Chu F. Signal optimization based generalized demodulation transform for rolling bearing nonstationary fault characteristic extraction. Mechanical Systems and Signal Processing 134 (2019): 106297.

[38] Liu H, Li J. A particle swarm optimization-based multiuser detection for receive-diversity-aided STBC systems. IEEE Signal Processing Letters 15 (2008): 29-32.

[39] Balafas K, Kiremidjian AS, Rajagopal R. The wavelet transform as a Gaussian process for damage detection. Structural Control and Health Monitoring 25.2 (2018): e2087.

[40] Gelman L, Soliński K, Ball A. Novel higher-order spectral cross-correlation technologies for vibration sensor-based diagnosis of gearboxes. Sensors, 20(2020), 1-23.

[41] Gelman L, Petrunin I, Parrish C, Walters M. Novel health monitoring technology for in-service diagnostics of intake separation in aircraft engines. Structural Control and Health Monitoring 27.5 (2020): e2479.

[42] Gelman L, Kırlangıç AS. Novel vibration structural health monitoring technology for deep foundation piles by non-stationary higher order frequency response function. Structural Control and Health Monitoring, 27(6) (2020) e2526. 